\title{
NEUROMYELITIS OPTICA LESION MIMICKING BRAINSTEM GLIOMA
}

A 12-year-old girl who presented with weakness of the left extremities and right sided sixth cranial nerve palsy had neuromyelitis optica (NMO) mistaken for brainstem glioma on MRI, in a report from Brain Research Institute, Yonsei University College of Medicine, Seoul, Republic of Korea. Right optic nerve atrophy diagnosed 10 months previously was unexplained. Admission MRI showed a right-sided pons lesion with high signal intensity on T2-weighted images, low signal intensity on T1-weighted images, and no enhancement after gadolinium. Following treatment with steroids and fractionated radiation, the left hemiparesis gradually improved, but a follow-up MRI showed no change in the size of the brainstem lesion. She was readmitted 2 months later with sudden loss of vision in the left eye and right arm paresthesiae in C3-4 dermatomes. Fundus exam revealed right optic atrophy and left optic neuritis. Brain MRI was unchanged. Spine MRI showed multiple signal changes at C-4 and T8-9 compatible with acute myelitis. CSF was normal and was negative for oligoclonal bands. MR spectroscopy showed a mildly elevated choline level, compatible with a demyelinating disease. Serum autoantibody marker NMO-IgG was positive. Immunosuppressive therapy with corticosteroids and azathioprine resulted in some improvement in vision in the left eye and marked reduction of paresthesiae. Plasmapheresis was of no benefit. Readmitted in respiratory distress 18 months later, the MRI showed lesions in the cervicomedullary junction. Despite artifical respiration the patient died of pulmonary failure. (Park KY, Ahn JY, Cho JH, Choi YC, Lee KS. Neuromyelitis optica with brainstem lesion mistaken for brainstem glioma. Case report. J Neurosurg (3 Suppl Pediatrics) September 2007;107:251-254). (Reprints: Jung Yong Ahn MD, PhD, Department of Neurosurgery, Yongdong Severance Hospital, 146-92, Do-gok-dong, Kangnam-gu, Seoul, 135-720, Rep of Korea. E-mail: jyahn@yumc.yonsei.ac.kr )

COMMENT. The diagnosis of NMO was suspected following the progression of neurological signs, 4 months after the initial presentation. The positive serum autoantibody NMO-IgG is diagnostic, with more than $90 \%$ specificity for patients with an optic-spinal syndrome. MR spectroscopy may be helpful in the differentiation of a demyelinating lesion from brainstem glioma. The mild elevation of choline in NMO is the result of increased levels of myelin breakdown products. In brainstem glioma, MR spectroscopy demonstrates decreased levels of $\mathrm{N}$-acetylaspartate and elevated levels of choline and creatine.

\section{HEADACHE DISORDERS}

\section{SUSTAINED BENEFIT OF 6-MONTH TOPIRAMATE TREATMENT FOR MIGRAINE}

The effects of discontinuing topiramate after a treatment period of 6 months in 818 patients with migraine, enrolled from 88 clinics in 21 countries in Europe, the UK and Turkey, are reported from University Duisburg-Essen, Germany. Patients were mean age 39.8 years, $13 \%$ male and $87 \%$ female. Patients received topiramate in a 26 -week open-label 
phase, after a 4-8 week lead-in period. Daily dose beginning at $25 \mathrm{mg}$ was increased in steps of $25 \mathrm{mg}$ every week to $100 \mathrm{mg}$. A further increase to $200 \mathrm{mg} / \mathrm{day}$ was made if required, but the dose remained stable for the last 4 weeks of treatment. Patients were randomly assigned to continue topiramate or switch to placebo for a 26-week double-blind phase. The number of days with migraine during the last 4 weeks of the double-blind phase was compared with the last 4 weeks of the open-label phase. The mean increase in number of migraine days was greater in the placebo group than in the topiramate group. Acute medication was used more frequently by the placebo than the topiramate group. Quality of life remained stable and patients were more satisfied with treatment efficacy while on topiramate compared to placebo. Tolerability was similar in both groups. Sustained benefit was obtained after discontinuing topiramate 6-month trial, but number of migraine days showed an increase. Patients should be treated for 6 months as a general rule, with the option to continue to 12 months in some patients. (Diener H-C, Agosti R, Allais G, et al. Cessation versus continuation of 6-month migraine preventive therapy with topiramate (PROMPT): a randomized, double-blind, placebo-controlled trial. Lancet Neurol December 2007;6:10541062). (Respond: Hans-Christoph Diener MD, Department of Neurology, University Duisburg-Essen, 45122 Essen, Germany).

COMMENT. This study of migraine prophylactic treatment included greater numbers of patients and was of longer duration than most previous trials. The authors hypothesize that the long-term effect of topiramate might be to correct the neuronal dysfunction factor in migraine pathophysiology. Since frequent migraine might lower the threshold for future attacks and promote development of chronic migraine, long-term prophylactic therapy should be considered as an option in adolescents with recurrent severe attacks.

The pros and cons of preventive compared to abortive antimigraine treatment are discussed in an editorial (Couch JR Jr. Preventive antimigraine therapy: the long-term outlook. Lancet Neurol Dec 2007;6:1035-1036). What are the indications for preventive therapy? Is the improvement worth the potential adverse effects? Does tachyphylaxis develop with long-term treatment? How quickly should treatment be withdrawn? Does preventive therapy lessen or increase the incidence of chronic daily headache? The results of the above study indicate that rapid withdrawal of topiramate caused a rebound headache occurrence, and future trials should lengthen the period of withdrawal. The data support the conclusion that preventive topiramate therapy has a residual beneficial effect, resetting the migraine cycle and raising the threshold.

\section{DEXAMETHASONE FOR ACUTE MIGRAINE}

A randomized trial of dexamethasone $10 \mathrm{mg}$ IV in 205 adult patients presenting with acute migraine to an emergency department (ED) found $25 \%$ of those with acute migraine became pain-free for at least 24 hours compared to $19 \%$ of those given placebo $(p=0.34)$. Patients with status migrainosus (migraine lasting longer than 72 hours) received persistent relief in $38 \%$ compared to $13 \%$ following placebo $(\mathrm{p}=0.06)$. All patients also received IV metoclopramide and diphenhydramine as pimary treatment for migraine. Researchers from the Department of Emergency Medicine (ED), Albert Einstein College of Medicine, Montefiore Medical Center, Bronx, NY, advise that IV dexamethasone should not be administered routinely in ED treatment of acute migraine, but it might be considered for 